\title{
PDE5 Inhibitor Tadalafil and Hydroxychloroquine Cotreatment Provides Synergistic Protection against Type 2 Diabetes and Myocardial Infarction in Mice
}

\author{
Rui Wang, Lei Xi, and Rakesh C. Kukreja \\ Pauley Heart Center, Division of Cardiology, Virginia Commonwealth University. Richmond, Virginia \\ Received November 18, 2016; accepted January 23, 2017
}

\begin{abstract}
Diabetes is associated with a high risk for ischemic heart disease. We have previously shown that phosphodiesterase 5 inhibitor tadalafil (TAD) induces cardioprotection against ischemia/ reperfusion (I/R) injury in diabetic mice. Hydroxychloroquine (HCQ) is a widely used antimalarial and anti-inflammatory drug that has been reported to reduce hyperglycemia in diabetic patients. Therefore, we hypothesized that a combination of TAD and $\mathrm{HCQ}$ may induce synergistic cardioprotection in diabetes. We also investigated the role of insulin-Akt-mammalian target of rapamycin (mTOR) signaling, which regulates protein synthesis and cell survival. Adult male $\mathrm{db} / \mathrm{db}$ mice were randomized to receive vehicle, TAD (6 mg/ $/ \mathrm{kg}), \mathrm{HCQ}(50 \mathrm{mg} / \mathrm{kg})$, or TAD + HCQ daily by gastric gavage for 7 days. Hearts were isolated and subjected to 30-minute global ischemia, followed by 1-hour reperfusion in Langendorff mode. Cardiac function and myocardial infarct size were determined. Plasma glucose, insulin and
\end{abstract}

lipid levels, and relevant pancreatic and cardiac protein markers were measured. Treatment with TAD $+\mathrm{HCQ}$ reduced myocardial infarct size $(17.4 \% \pm 4.3 \%$ vs. $37.8 \% \pm 4.9 \%$ in control group, $P<0.05)$ and enhanced the production of ATP. The TAD + HCQ combination treatment also reduced fasting blood glucose, plasma free fatty acids, and triglyceride levels. Furthermore, TAD + HCQ increased plasma insulin levels (513 \pm 73 vs. $232 \pm$ $30 \mathrm{mU} /$ liter, $P<0.05$ ) with improved insulin sensitivity, larger pancreatic $\beta$-cell area, and pancreas mass. Insulin-like growth factor-1 (IGF-1) levels were also elevated by TAD + HCQ (343 \pm 14 vs. $262 \pm 22 \mathrm{ng} / \mathrm{ml}, P<0.05)$. The increased insulin/lGF-1 resulted in activation of downstream Akt/mTOR cellular survival pathway. These results suggest that combination treatment with TAD and HCQ could be a novel and readily translational pharmacotherapy for reducing cardiovascular risk factors and protecting against myocardial I/R injury in type 2 diabetes.

\section{Introduction}

Worldwide, type 2 diabetes (T2D) is one of the major risk factors for developing cardiovascular disease and the resultant devastating morbidity and mortality (Ginter and Simko, 2012; Nichols et al., 2013). Diabetic patients with myocardial infarction have a worse prognosis than do nondiabetic patients with myocardial infarction (Miettinen et al., 1998). In diabetic hearts, reduced glucose uptake and increased circulating free fatty acids lead to a shift of energy substrate from carbohydrates to fatty acids, resulting in less ATP production with more oxygen consumption, which makes cells more susceptible to myocardial ischemia (Barsotti et al., 2009). Recently, it has been suggested that T2D hearts retain metabolic flexibility to adapt to hypoxia and become more dependent on oxidative metabolism after hypoxia, with $30 \%$ lower glycolytic rates and

This study was supported by the National Institutes of Health, National Heart, Lung, and Blood Institute [Grants R01HL118808 and R37HL51045] to R.C.K

This work was previously presented as a poster presentation at the following meeting: Diabetes, Obesity and Cardiovascular Health: Insights from Basic Science. American Heart Association Scientific Sessions; 2015 Nov 8; Orlando, FL. Session No. APS.78.03, American Heart Association, Dallas.

dx.doi.org/10.1124/jpet.116.239087
$36 \%$ higher fatty acid oxidation than nondiabetic controls, which leads to a functional deficit in response to ischemic stress (Mansor et al., 2016). Thus, normalization of circulating glucose and lipid levels is a critical target of therapeutic intervention in the treatment of patients with T2D (Nichols et al., 2013; Fukushima et al., 2015; Gilbert and Krum, 2015).

Phosphodiesterase 5 (PDE5) inhibitors are a class of drugs widely used to treat erectile dysfunction. Two of the PDE5 inhibitors, sildenafil and tadalafil (TAD), are also approved by the Food and Drug Administration for the treatment of pulmonary arterial hypertension (Hemnes and Champion, 2006; Galie et al., 2009). In addition, several studies from our laboratory have shown that PDE5 inhibitors protect against myocardial ischemia/reperfusion (I/R) injury in both healthy and diabetic animals (reviewed in Das et al., 2015). The underlying molecular mechanism involves the induction of nitric oxide synthase (Salloum et al., 2003; Das et al., 2005), which leads to subsequent nitric oxide-cGMP-protein kinase G-signaling cascade with promotion of hydrogen sulfide production (Salloum et al., 2009), activation of extracellular signal-regulated kinase 1/2 pathway (Das et al., 2009), and opening of mitochondrial $\mathrm{K}_{\text {AтP }}$ channels (Ockaili et al., 2002). Our previous studies showed that 4 -week chronic treatment of $\mathrm{db} / \mathrm{db}$ mice with

ABBREVIATIONS: CTRL, control; ELISA, enzyme-linked immunosorbent assay; HCQ, hydroxychloroquine; IGF-1, insulin-like growth factor-1; I/R, ischemia/reperfusion; mTOR, mammalian target of rapamycin; OGT, oral glucose tolerance test; PDE5, phosphodiesterase 5; T2D, type 2 diabetes; TAD, tadalafil; TTC, 2,3,5 triphenyltetrazolium chloride. 
long-acting PDE5 inhibitor TAD resulted in a reduction of blood glucose and triglyceride levels and smaller myocardial infarct size (Varma et al., 2012), indicating beneficial effects in maintaining homeostasis of whole-body metabolism and protecting against I/R injury under T2D conditions.

Hydroxychloroquine (HCQ) is a first-line antimalarial and anti-inflammation drug that has been widely used to treat malaria, systemic lupus erythematosus, and rheumatoid arthritis (Morand et al., 1992; Anderson, 1995). Chloroquine has been reported to improve insulin sensitivity through the activation of Akt, resulting in increased glucose uptake and glycogen synthesis in L6 muscle cell lines (Halaby et al., 2013). Also, clinical case reports indicated that both chloroquine and HCQ improved glycemic control in patients with type 1 diabetes, partially through the inhibition of insulin degradation (Blazar et al., 1984; Hage et al., 2014). A 3-day oral chloroquine treatment improved the serum lipid profile of T2D patients with increased fasting insulin level (Powrie et al., 1993). HCQ users among rheumatoid arthritis patients had a lower risk of developing diabetes (Bili et al., 2011), suggesting a protective effect of HCQ against insulin resistance; however, whether this antidiabetic effect of HCQ could further lead to cardioprotection against I/R injury, the most common cause of death in T2D, is unknown. One study reported that oral administration of $200 \mathrm{mg} / \mathrm{kg}$ HCQ for 3 days before I/R injury significantly decreased myocardial infarct size in nondiabetic rats (Bourke et al., 2015).

Based on this background information, we hypothesized that TAD in combination with HCQ could synergistically protect $\mathrm{T} 2 \mathrm{D} \mathrm{db} / \mathrm{db}$ mouse heart from I/R injury through mechanisms involving insulin-Akt/mTOR pathway and modulation of circulatory energy substrate levels. We also investigated the effects of TAD and HCQ alone and in combination on the islets of Langerhans in the pancreas.

\section{Materials and Methods}

Animals. All animal experiments were conducted under the guidelines on humane use and care of laboratory animals for biomedical research published by the National Institutes of Health (no. 85-23, Revised 1996). The animal care and experimental protocols were approved by the Institutional Animal Care and Use Committee of Virginia Commonwealth University. Adult male db/db mice (B6. BKS(D)-Lepr ${ }^{d b} / \mathrm{J}$ ) were obtained from Jackson Laboratory (Bar Harbor, ME). Mice with a fasting glucose level between 200 and $400 \mathrm{mg} / \mathrm{dl}$ were included in the experiments.

Drugs and Treatment Protocols. Seventy-six male $\mathrm{db} / \mathrm{db}$ mice were assigned into four groups ( $n=19$ /group) and received the following treatments via daily oral gavage for 7 days: group 1, control vehicle (CTRL, $0.5 \%$ hydroxypropyl methy cellulose and $1 \%$ Tween 80 in sterile water); group 2, TAD (Lilly USA, Indianapolis, IN), $6 \mathrm{mg} / \mathrm{kg}$ daily mixed in vehicle solvent; group 3, HCQ (Plaquenil, Sanofi-Aventis U.S., Bridgewater, NJ) $50 \mathrm{mg} / \mathrm{kg}$ daily mixed in vehicle solvent; and group 4, TAD (6 mg/kg daily) + HCQ (50 mg/kg daily) mixed in vehicle solvent. The oral dose of HCQ was chosen based on a recent study (Long et al., 2013), which used $50 \mathrm{mg} / \mathrm{kg}$ daily in adult rats administered by i.p. injection for 3 weeks. Chronic treatment with this dose of HCQ provided significant beneficial effects against pulmonary hypertension. In addition, this experimental dose of $\mathrm{HCQ}$ is compatible with the clinically prescribed dose (i.e., $250 \mathrm{mg}$ per day; $\sim 3.8 \mathrm{mg} / \mathrm{kg}$ for an adult with 65 -kg body weight) for the treatment of rheumatoid arthritis and systemic lupus erythematosus. According to the Food and Drug Administration, the human HCQ dose $(250 \mathrm{mg} / \mathrm{kg}$ day) can be converted into mouse dose (i.e., human dose multiple by
$12.5=50 \mathrm{mg} / \mathrm{kg}$ daily). The final numbers of animals used for data analysis are $n=19$ for CTRL, TAD, and HCQ group respectively, $n=17$ for TAD + HCQ group (two TAD + HCQ-treated mice died apparently from physical injury during the oral gavage procedure). Body weight was recorded, and fasting blood glucose levels were measured using Bayer Contour blood glucose monitoring system (Bayer, Leverkusen, Germany).

After completion of the 1-week treatment, animals were anesthetized with i.p. injection of pentobarbital sodium $(100 \mathrm{mg} / \mathrm{kg})$ and euthanized. Their hearts and pancreata were surgically collected. A subset of hearts were subjected to I/R in the Langendorff model $(n=$ 5 for TAD, HCQ, and TAD + HCQ groups and $n=6$ for the CTRL group), and the tissue samples from other animals were snap-frozen in liquid nitrogen and stored at $-80^{\circ} \mathrm{C}$. Meanwhile, blood samples were collected from all animals into heparinized tubes, and plasma was separated by centrifugation at $4^{\circ} \mathrm{C}, 3000 \mathrm{rpm}$ for 30 minutes.

I/R Studies. The I/R studies were performed in isolated perfused hearts using the Langendorff model as described previously (Xi et al., 1999). In brief, after the animals were anesthetized, the hearts were isolated and immediately immersed into $95 \%$ oxygen $/ 5 \% \mathrm{CO}_{2}$ saturated ice cold Krebs-Henseleit buffer, containing (in $\mathrm{mM}$ ) $118 \mathrm{NaCl}$, $24 \mathrm{NaHCO}_{3}, 4.7 \mathrm{KCl}, 1.2 \mathrm{KH}_{2} \mathrm{PO}_{4}, 1.2 \mathrm{MgSO}_{4}, 11 \mathrm{D}$-(+)-Glucose, 0.5 EDTA, and $2.5 \mathrm{CaCl}_{2}$. Within 3 minutes, the aorta was cannulated and mounted onto a Langendorff perfusion system and the hearts were retrogradely perfused with Krebs-Henseleit buffer and maintained at $37^{\circ} \mathrm{C}$ under a constant pressure of $55 \mathrm{~mm} \mathrm{Hg}$. The heart apex was attached to a Grass FT03 force-displacement transducer (Grass Technologies, West Warwick, RI) with surgical thread and a rigid metal hook for measuring ventricular contractile force. The beat-bybeat cardiac contractile signals were continuously recorded with a PowerLab/8sp system (ADInstruments Inc., Colorado Springs, CO). After 30 minutes of equilibration, hearts were subjected to 30 minutes of normothermic no-flow global ischemia $\left(37^{\circ} \mathrm{C}\right)$, followed by 1 hour of reperfusion. Coronary flow rate was measured via timed collection of coronary effluents at 15 minutes before ischemia and 15 minutes after reperfusion. At the end of the experiment, the hearts were collected, weighed, and stored at $-20^{\circ} \mathrm{C}$ for further analysis.

Infarct Size Measurement. The day after the Langendorff experiment, frozen hearts were transversely cut into six or seven sections, $1 \mathrm{~mm}$ thick, and placed in Petri dishes. The heart sections were stained with $10 \%$ 2,3,5 triphenyltetrazolium chloride (TTC, Sigma-Aldrich, St. Louis, MO) for 30 minutes and then transferred into $10 \%$ neutral-buffered formalin for fixation (Sigma-Aldrich). After taking pictures of each section, the infarct size was quantified by computerized morphometry using ImageJ software National Institutes of Health, Bethesda, MD). The infarct size was presented as the percentage of risk area (i.e., the total ventricular area minus cavities). A portion of the heart tissue was used for preparation of protein extracts.

Insulin, Insulin-Like Growth Factor, and Lipid Assays. Plasma insulin levels were determined using an insulin enzymelinked immunosorbent assay (ELISA) kit (Crystal Chem Inc., Downers Grove, IL). Insulin-like growth factor-1 (IGF-1) levels were measured in plasma samples using mouse/rat IGF-1 Quantikine ELISA (R\&D systems, Minneapolis, MN). Nonesterified fatty acids, triglycerides, and cholesterol levels were assayed using nonesterified fatty acids, L-Type Triglyceride M and Cholesterol E assay kits (Wako Pure Chemical Industries, Osaka, Japan). The cardiac levels of triglycerides and total cholesterol were normalized with total protein content.

Glucose and Insulin Tolerance Test. The glucose tolerance test was performed in mice after overnight fasting. After measurement of body weight, each animal received glucose ( $2 \mathrm{mg} / \mathrm{kg}$ body weight) via oral gavage ( $n=5 /$ group). Blood samples were drawn from the tail vein at baseline, $15,30,60$, and 120 minutes after glucose administration. The plasma glucose and insulin levels were measured by Glucose Colorimetric Assay Kit (Cayman Chemical, Ann Arbor, MI) and insulin ELISA Kit (Crystal Chem Inc., Downers Grove, IL). For the insulin tolerance test (ITT), the animals ( $n=5$ /group) were fasted 
for 6 hours. After measuring body weight, regular human insulin (Novolin, Novo Nordisk, Princeton, NJ) was administered intraperitoneally $(0.9 \mathrm{IU} / \mathrm{kg})$. Blood glucose levels were taken at baseline, 15 , $30,60,90$, and 120 minutes after insulin injection, and glucose levels were measured using a Bayer Contour blood glucose monitor.

Immunofluorescence and H\&E Histology. Mouse pancreata were weighed, and the ratio of pancreas weight versus body weight was calculated ( $n=5$ /group). Immunohistology was performed according to a previous published method, with modifications (Kim et al., 2011). Briefly, the mouse pancreata were fixed in $10 \%$ neutralbuffered formalin and embedded in paraffin. Tissue was sectioned to a thickness of $5 \mu \mathrm{m}$. Multiple sections (200 $\mu \mathrm{m}$ apart) from each animal were chosen and stained with goat anti-insulin (Santa Cruz Biotechnology, Dallas, TX), followed by secondary antibody conjugated with fluorescein isothiocyanate (Life Technology). Images of insulinpositive $\beta$ cells were taken under microscope, and cross-sectional areas of insulin positive $\beta$ cells, as well as relative area of pancreas, were determined. The insulin-positive $\beta$-cell area/pancreas area was calculated. Also, sections of pancreas were stained with $\mathrm{H} \& \mathrm{E}(n=8$ for TAD and $n=9$ for CTRL, HCQ, and TAD + HCQ). Whole sections were scanned with a NanoZoomer 2.0-HT Digital slide scanner (Hamamatsu, Japan), and cross-sectional islet numbers were counted. The number of islets per $\mathrm{mm}^{2}$ tissue area was calculated.

Western Blot Analysis for Protein Expression. The heart tissue was crushed in liquid nitrogen, transferred into RIPA buffer (Thermo Scientific Pierce no. PI-89900), and further homogenized. Samples were then centrifuged at $4^{\circ} \mathrm{C}, 12,000 \mathrm{rpm}$, for 10 minutes, and supernatant was collected. Protein concentration was determined using BCA reagent (no. 23227; Thermo Fisher Scientific, Somerset, NJ). Samples were then mixed with $2 \times$ sample buffer (no. 1610737; Bio-Rad Laboratories, Hercules, CA) and boiled for 5 minutes before loading on $4 \%-15 \%$ Criterion TGX Gel system (30 $\mu \mathrm{g} / \mathrm{sample}$ ) for electrophoresis. A wet transfer was performed using Bio-Rad Criterion blotter system. Membranes were then incubated in 5\% nonfat milk for 1 hour at room temperature, followed by incubation with primary antibodies (1:1000 dilution) at $4^{\circ} \mathrm{C}$ overnight. The membranes were further incubated with secondary antibodies and then developed using $20 \times$ LumiGLO Reagent and $20 \times$ peroxide (no. 7003; Cell Signaling Technology, Danvers, MA). We chose $\beta$-actin as the internal reference. Antibodies used were rabbit anti-p-mTOR no. 5536, rabbit anti-mTOR no. 2938, rabbit anti-p-S6 no. 4858, mouse antiS6 no. 2317 , rabbit anti-pAkt ${ }^{\text {Thr308 }}$ no. 4056 , rabbit anti-p-Akt ${ }^{\text {Ser473 }}$ no. 4060 , rabbit anti-Akt no. 4691, rabbit anti-Raptor no. 2280 , rabbit anti-Rictor no. 2114 (Cell Signaling), and mouse anti- $\beta$-actin sc-58679 (Santa Cruz Biotechnology).

ATP Assay. Frozen myocardial tissue samples (10 mg from each animal, $n=5$ /group) were used to quantify ATP levels in the tissue using ATP Assay Kit (Abcam Biotechnology, Cambridge, UK) according to the manufacturer's instructions.

Statistics. Data were analyzed using GraphPad Prism 6 software (GraphPad Software, Inc., La Jolla, CA). One-way analysis of variance and Dunnett's post hoc test were performed for global and pairwise comparisons of the multiple groups. Data were presented as mean \pm S.E.M. and $P<0.05$ was considered statistically significant.

\section{Results}

Treatment with Tadalafil and Hydroxychloroquine Reduces Myocardial Infarct Size after I/R Injury. The experimental protocol is shown in Fig. 1. Mice treated with the combination of TAD and HCQ had a significantly smaller myocardial infarct size as shown by TTC staining (Fig. 2). After 7 days of the drug treatment, no difference was observed in cardiac contractile function based on ventricular developed force (Fig. 3A) and rate-force product (Fig. 3B) before ischemia and at the end of reperfusion. Heart rate (Fig. 3C) and

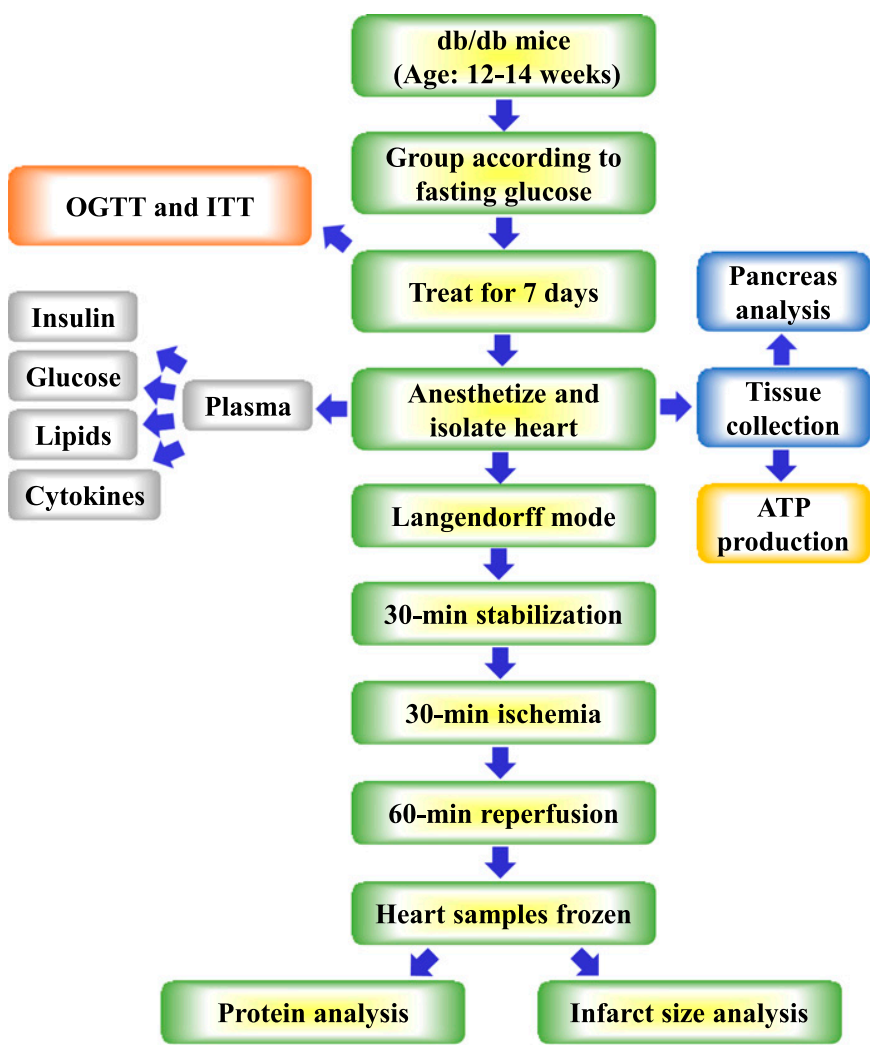

Fig. 1. Experimental design and protocol. The schematic diagram shows the sequence and timing of various experimental procedures, including the drug treatment, OGTT, insulin tolerance test (ITT), harvest of tissue, global I/R in Langendorff mode, histology analysis, as well as plasma and cardiac tissue biochemical measurements.

coronary flow rate (Fig. 3D) also did not differ among the treatment groups.

Effect of Tadalafil and Hydroxychloroquine Treatment on Blood Glucose, Insulin, IGF-1, and Lipids. Baseline levels of fasting glucose were measured before any treatment.

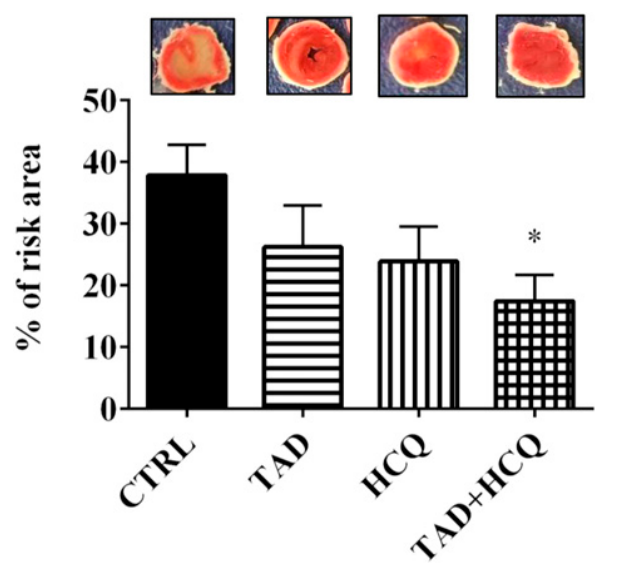

Fig. 2. Effect of TAD, HCQ, and combination treatment on postischemic infarct size in $\mathrm{db} / \mathrm{db}$ mice. Top: Representative images of transverse sections of TTC-stained hearts collected after 1-week respective drug treatments and ex vivo global I/R. Bottom: Bar diagram showing myocardial infarct size presented as $\%$ of risk area (mean \pm S.E.M., $n=$ 6 for CTRL and $n=5$ for TAD, HCQ, and TAD + HCQ). $* P<0.05$ versus CTRL group. 

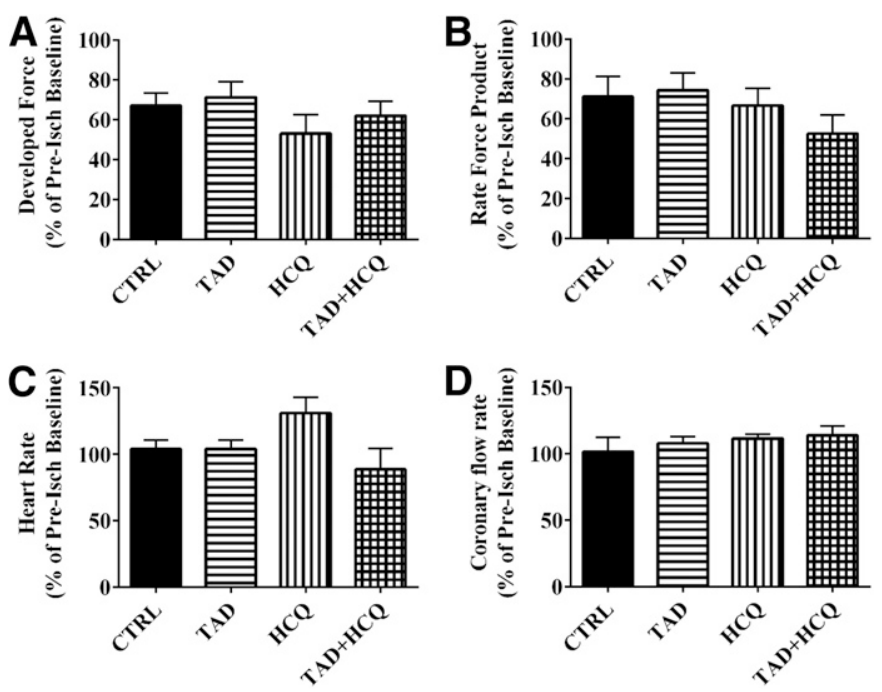

Fig. 3. Effect of TAD, HCQ, and combination treatment on cardiac function. Post-I/R ventricular developed force (A), rate-force product (B), heart rate (C), and coronary flow rate (D) are presented as \% of preischemia baseline (mean \pm S.E.M.; $n=6$ for CTRL and $n=5$ for TAD, HCQ, and TAD + HCQ). No statistical significance was observed among the four treatment groups in any of the cardiac function indices.

The plasma level of insulin was increased after 7 days of treatment with HCQ alone or in combination with TAD compared with the control group (Fig. $4 \mathrm{~A}, P<0.05$ ). The same trend was observed in the TAD-treated group as well. IGF-1 levels increased only in the TAD + HCQ combination treatment group (Fig. 4B). The blood glucose level increased significantly from the baseline level in the control group; however, no such increase in glucose levels was observed in TAD, HCQ, or especially the TAD + HCQ group, which had a significantly lower glucose levels compared with the control group after 1 week of treatment (Fig. 4C). Lipid profile was also altered after TAD + HCQ treatment, with a significant decrease in the plasma levels of free fatty acids and triglycerides in the TAD + HCQ group (Fig. 4, D and $\mathrm{E}$ ). There was a trend toward decreased cholesterol levels in all three drug-treated groups, although the differences did not reach statistical significance (Fig. 4F). Interestingly, cardiac levels of triglycerides increased in HCQ-treated group (Fig. 4G), whereas cholesterol levels in the combination treated hearts were decreased (Fig. 4H).

\section{Beneficial Effects of the Combination Therapy with Tadalafil and Hydroxychloroquine on Insulin Sensitivity}

We performed oral glucose tolerance test (OGTT) and ITT to assess the effect of combination treatment of TAD and HCQ on insulin sensitivity in the $\mathrm{db} / \mathrm{db}$ mice. The results showed no significant difference in glucose uptake or insulin release between the control and TAD + HCQ groups during OGTT (Fig. 5, A-D); however, TAD + HCQ treatment group showed a larger decline in blood glucose levels in response to insulin injection (Fig. 5, E- G), as indicated by approximately $20 \%$ drop in the area under the curve (Fig. 5F), suggesting improved insulin sensitivity.

Tadalafil and Hydroxychloroquine Increase Pancreatic $\boldsymbol{\beta}$-Cell Area. We next asked whether the observed increase in fasting insulin levels (Fig. 4A) was due to a protective effect of the drug treatment on the pancreatic $\beta$-cells. Immunofluorescence staining showed that the total insulin positive $\beta$-cell area was markedly increased after treatment with either TAD or HCQ alone and in combination (Fig. 6, A and C). There was a significant increase in the islet number per $\mathrm{mm}^{2}$ in the pancreas (Fig. 6, B and D). In addition, TAD-, HCQ-, and TAD + HCQ-treated animals had a clear trend toward increased pancreas mass/body weight; the increase in the HCQ and TAD + HCQ groups was statistically significant (Fig. 6E).

Effect of Tadalafil and Hydroxychloroquine on Cardiac Insulin/IGF-1-Akt-mTOR Pathway. Insulin/ IGF-1 triggers downstream Akt-mTOR pathway in response to food and energy intake to stimulate glucose uptake and glycogen and protein synthesis. We observed increased Akt phosphorylation at site $\mathrm{Thr}^{308}$ after I/R in mice treated with TAD, HCQ, and TAD + HCQ combination (Fig. 7, A and B). As a result, the downstream mammalian target of rapamycin (mTOR) was activated. Phosphor-mTOR was increased by the combination treatment (Fig. 7, A and C). We also investigated if both (mTORC1) and mTORC2 were activated by drug treatments. The expression of mTORC1 protein Raptor was increased in all three drug-treated groups after I/R (Fig. 7, A and D), which was associated with enhanced phosphorylation of $\mathrm{S} 6$, the mTORC1 downstream ribosomal protein (Fig. 7, A and $\mathrm{E})$. The total $\mathrm{S} 6$ protein expression was also increased in TAD-, HCQ-, and TAD + HCQ-treated groups (Fig. 7, A and $\mathrm{E})$. The mTORC2 protein Rictor expression was also upregulated in TAD-, HCQ-, and TAD + HCQ-treated hearts (Fig. 7, $\mathrm{A}$ and F). Consequently, phosphor-Akt ${ }^{\text {Ser473 }}$, the downstream target of $\mathrm{mTORC} 2$ was dramatically increased in the HCQ and TAD + HCQ treatment groups (Fig. 7, A and G).

TAD + HCQ Combination Treatment Improved ATP Levels in Heart. To determine whether the beneficial effects of TAD and HCQ on cardiac cell survival, circulating insulin/ IGF-1, or the blood profile of lipids are correlated with the improvement in the final product of cardiac energy metabolism, we also measured ATP level in the heart. Our results showed that both HCQ and TAD + HCQ groups had significantly increased levels of cardiac ATP production after 7-day treatment compared with TAD- or HCQ-treated groups $(P<$ 0.01, Fig. 8).

\section{Discussion}

The main features of T2D are hyperglycemia, hyperlipidemia, insulin resistance, and reduced insulin production in the late stage. It has been well recognized that diabetic individuals are resistant to most of the cardioprotective drugs that would be beneficial in the nondiabetic population. The pathology of myocardial I/R injury involves multiple pathways, which include calcium overload, $\mathrm{pH}$ paradox, generation of reactive oxygen species, inflammation, endothelial dysfunction, and altered myocardial metabolism (Yellon and Hausenloy, 2007). Numerous therapeutic strategies targeting single mechanistic pathway have limited effect on human and animal models, indicating that myocardial $\mathrm{I} / \mathrm{R}$ injury is a complex confluence of divergent biologic signaling.

Our previous studies reported that after 4 or 8 weeks of treatment, TAD provided myocardial protection in $\mathrm{db} / \mathrm{db}$ mice by improving mitochondrial function, increasing NO bioavailability, and inhibiting inflammation (Varma et al., 2012; Koka et al., 2014). In addition, in vivo, in vitro, and cohort-based reports have shown that HCQ has multiple other properties that include beneficial effects on metabolism function, vascular 

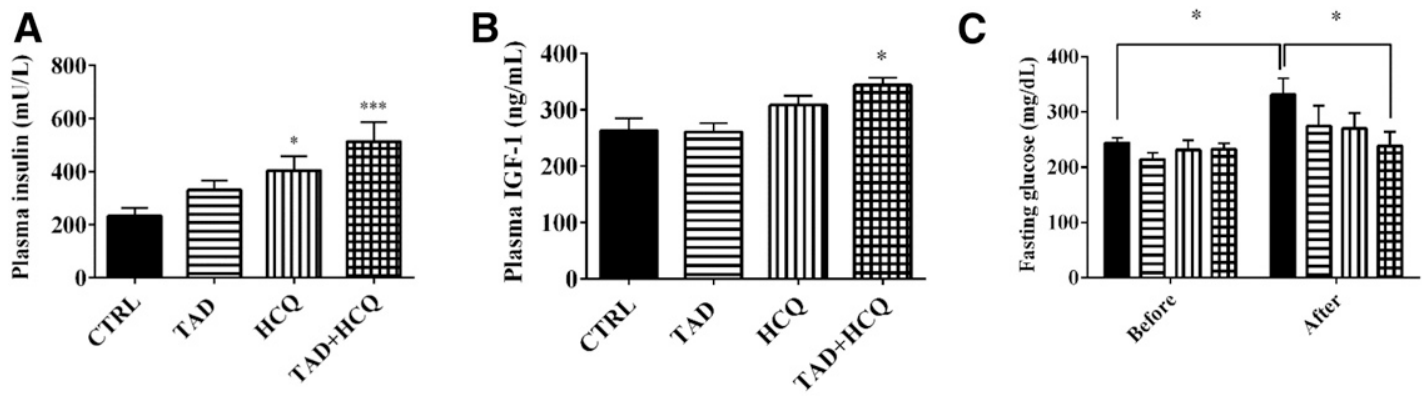

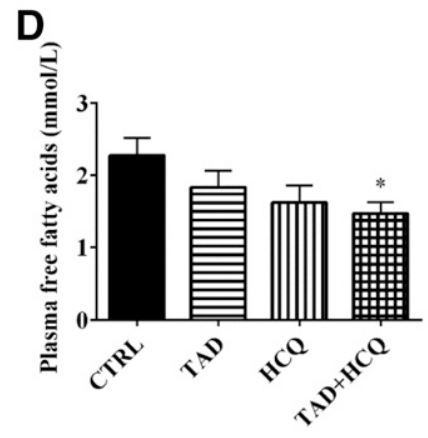

$\mathbf{E}$
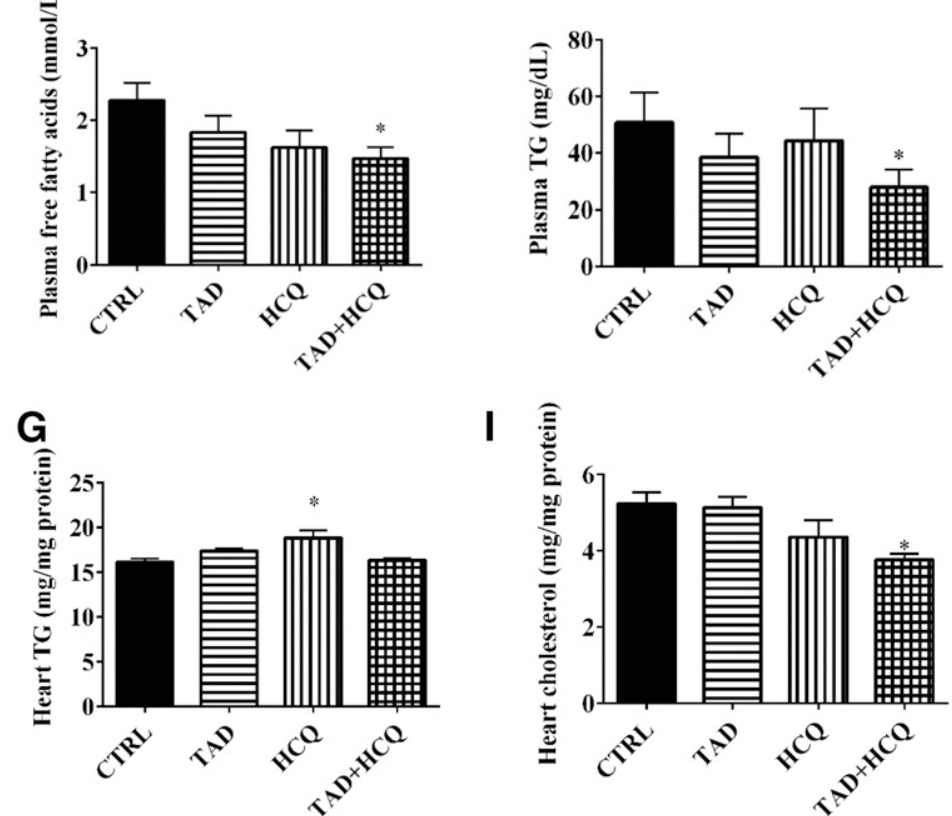

I

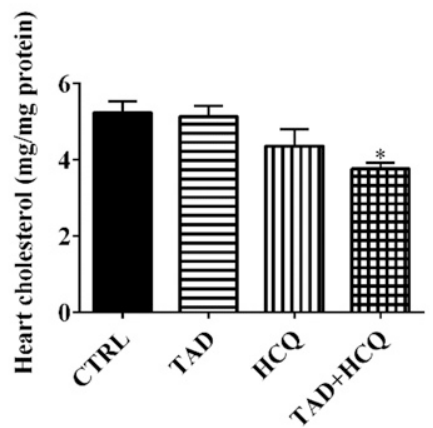

Fig. 4. Changes in blood glucose, plasma insulin, IGF-1 levels, and lipid profile after treatment with TAD and HCQ. (A, B) Plasma insulin and IGF-1 levels measured with ELISAs. (C) Blood glucose levels measured before and after the 1-week drug treatment, respectively (mean \pm S.E.M.; $n=13$ for CTRL and HCQ, $n=15$ for TAD, and $n=12$ for TAD + HCQ). (D-H) Plasma and cardiac levels of free fatty acids, triglycerides, and total cholesterol measured using enzymatic assays (mean \pm S.E.M., $n=8$ for TAD, $n=9$ for $\mathrm{HCQ}$, and $n=12$ for CTRL and TAD + HCQ). *P< 0.05 ; *** $P<0.001$ versus CTRL group.

compliance, and endothelial function (Blazar et al., 1984; Powrie et al., 1993; Siso et al., 2008; Bili et al., 2011; Halaby et al., 2013; Hage et al., 2014). In the present study, we report a novel and potentially important combination use of TAD and HCQ in protection against myocardial $\mathrm{I} / \mathrm{R}$ injury and reduction of cardiovascular risk factors (e.g., hyperglycemia and hyperlipidemia) in T2D. This novel strategy of combining both TAD and HCQ provided superior beneficial effects than a monotherapy in T2D. The short-term treatment of 7 days with TAD and HCQ resulted in improved blood glucose, free fatty acids, triglyceride levels, and improved insulin signaling, which ultimately contributed to significant reduction of infarct size; however, the improvement in cardiac function was not evident after the drug treatment (Fig. 3). Such dissociation between the infarct size reduction and improvement of contractile function is not unusual because previously published studies by our group and others have also observed that ischemic preconditioning (Jenkins et al., 1995; Xi et al., 1998) or stem cell therapy (Moelker et al., 2006) causes a significant reduction in infarct size without concomitant improvement in ventricular function. A possible explanation for this dissociation is that there may be two separate mechanisms controlling cardiac cell survival and contractility, respectively. A protective mechanism on cardiomyocyte viability may not necessarily translate into a better contractility or vice versa. The phenomenon of myocardial stunning is another example in which cardiomyocytes are viable, but their contractility is severely depressed. The experimental and clinical studies have also shown that myocardium reperfused after reversible ischemia exhibits prolonged depression of contractile function (Bolli et al., 1991). Furthermore, the viable but stunned myocardium can gradually regain its contractility, but infarcted myocardium would likely to have sustained contractile dysfunction. Therefore, the infarct size reduction by TAD + HCQ therapy may eventually lead to a better cardiac function, which needs to be examined in a larger in vivo translational model of T2D after prolonged reperfusion.

In diabetic patients, increased free fatty acids are released from adipose tissue through triglyceride lipolysis (Stich and Berlan, 2004), leading to higher circulating free fatty acid levels that can further result in increased $\beta$-oxidation and reduction of glycolysis in cardiomyocytes. The combination treatment of TAD and HCQ reduced plasma free fatty acids and blood glucose levels, as well as cardiac cholesterol content, suggesting a myocardial protective effect through regulation 

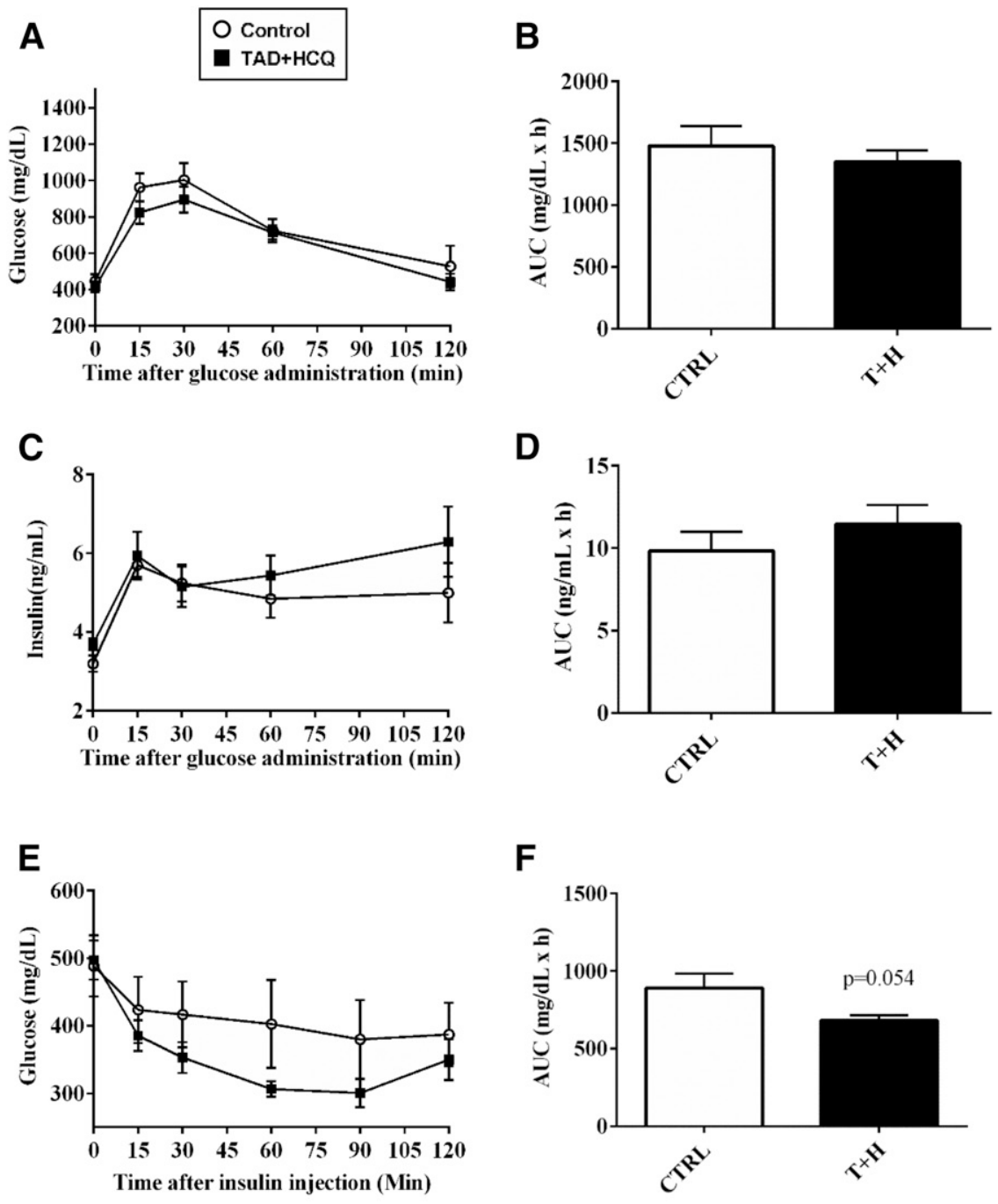

Fig. 5. Insulin tolerance test (ITT) and OGTT after treatment with TAD, HCQ, and a combination of TAD + HCQ. Animals were fasted overnight before the OGTT. Glucose $(2 \mathrm{mg} / \mathrm{kg})$ was administered via oral gavage, and blood samples were taken from the tails. (A) Glucose levels; (B) area under the curve; (C) insulin levels; and (D) area under the curve. The ITT was performed after the animals were fasted for 6 hours. Insulin $(0.9 \mathrm{IU} / \mathrm{kg}$ regular human) was given i.p.; (E) blood glucose levels; (F) area under the curve; $(\mathrm{G})$ insulin response curve is presented as percentage to baseline glucose. Data are mean \pm S.E.M. $(n=5 /$ group,). $* P<0.05$ versus CTRL group.

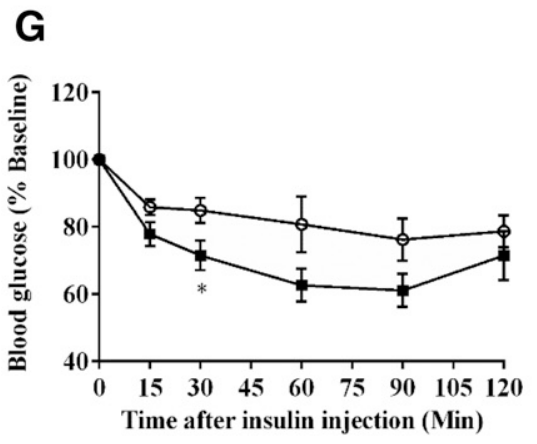

of both circulating energy substrates and cardiac lipids (Fig. 4, C-H). Previous study showed that higher cardiac cholesterol content in the hypercholesterolemic rats was associated with the loss of sevoflurane-induced cardioprotection against I/R injury via alteration of the survival kinase signaling pathway (Xu et al., 2013). In addition, hesperidin-induced protection against isoproterenol-induced cardiotoxicity was associated with a reduction in cardiac cholesterol levels (Selvaraj and Pugalendi, 2012). Accordingly we postulate that the observed decrease in cardiac cholesterol content in the TAD + HCQ-treated diabetic mice (Fig. 4H) may also play a cardioprotective role against I/R injury.
In the present study, we observed increased baseline insulin levels in animals treated with combination of TAD and HCQ. Also, better insulin sensitivity was observed, with improved insulin response 30 minutes after insulin injection (Fig. 5). Furthermore, the mass of pancreas was larger in $\mathrm{HCQ}$, as well as TAD+HCQ-treated groups, with increased insulin-positive $\beta$-cell area in the latter group (Fig. 6). The pancreas weight/ body weight was higher in HCQ- and the combination-treated groups, suggesting a possible protective effect on $\beta$ cells as well. These results support previous clinical studies showing improvement in $\beta$-cell function with HCQ or TAD in human subjects (Hill et al., 2009; Wasko et al., 2015). Interestingly, 


\section{A}
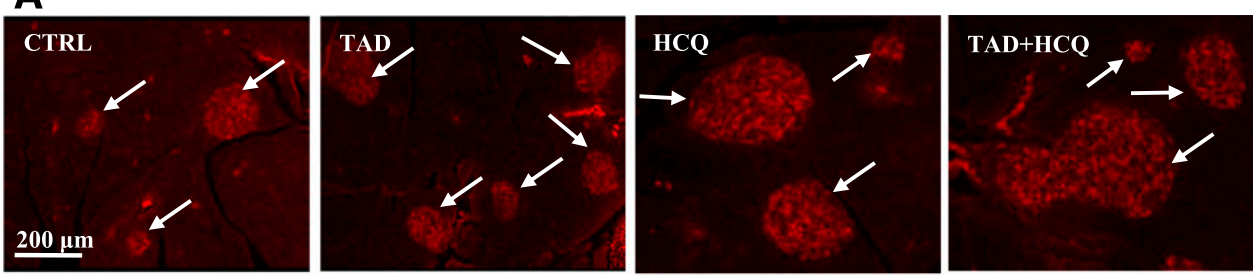

B
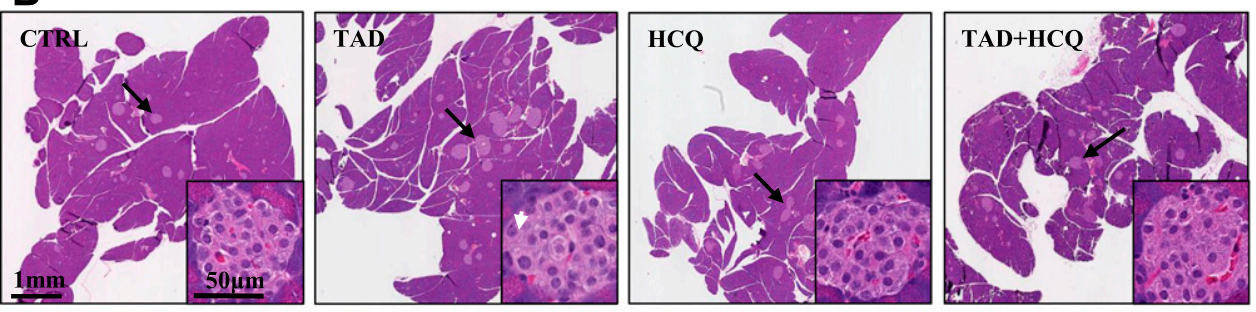

C

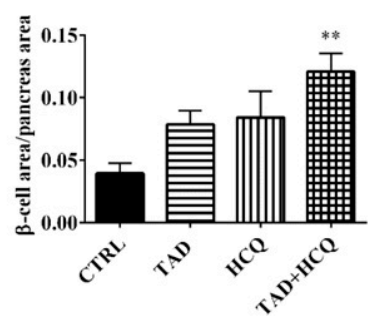

D
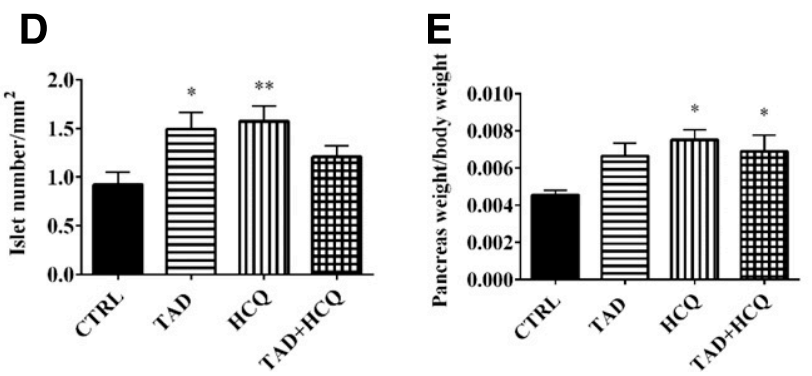

Fig. 6. Effect of TAD, HCQ, or combination treatment on pancreatic islets. (A) Representative pictures of immunofluorescent-stained paraffin sections of pancreata. Goat anti-insulin antibody was used to detect insulin inside islets, and a secondary antibody conjugated with fluorescein isothiocyanate was used. (B) Representative pictures of H\&E-stained pancreas, paraffin-fixed sections, with further magnified representative images of the pancreatic islets at lower-right corners. (C) Insulin-positive $\beta$-cell area versus pancreas area ( $n=5$ /group). (D) Bar diagram showing pancreatic islet number per $\mathrm{mm}^{2}$ pancreas area in all treatment groups ( $n=8$ for TAD; $n=9$ for CTRL, $\mathrm{HCQ}$, and TAD + HCQ). (E) Bar diagram showing the percentage of pancreas mass versus body weight ( $n=5$ /group). Data are mean \pm S.E.M. $* P<0.05 ; * * P<0.01$ versus CTRL. there was no significant difference in the insulin release during the OGTT test, which suggests that the combination treatment is effective only in improving baseline (fasting) insulin secretion.

We also observed higher plasma IGF-1 levels in the combination treatment group, which possibly leads to activation of PI3K/Akt/mTOR pathway in $\beta$-cells. It has been shown that IGF-1 promotes pancreatic $\beta$-cell line INS-1 cell proliferation in vitro within the physiologically relevant glucose concentration (6-18 $\mathrm{mM})$. This synergistic effect induces more than 50-fold cell proliferation (Hugl et al., 1998); however, other studies have reported that pancreatic specific IGF-1 knockout mice had a 2.3-fold increase in pancreatic cell mass, suggesting that locally produced IGF-I within the pancreas inhibits islet cell growth ( $\mathrm{Lu}$ et al., 2004). In addition, $\beta$-cell- specific IGF-1 receptor knockout mice showed normal growth and development of $\beta$ cells but had a defective glucose-stimulated insulin secretion with impaired glucose tolerance (Kulkarni et al., 2002). Thus, the function of IGF-1 in $\beta$-cell proliferation remains inconclusive.

Insulin and IGF-1 are well known to control blood glucose levels (Guler et al., 1987). The increased levels of insulin and IGF-1 play an important role in improving hyperglycemia. Also, both insulin and IGF-1 bind to insulin receptors and IGF-1 receptors with different affinity, regulating cell survival through activation of the PI3K-Akt pathway (Buerke et al., 1995; Jonassen et al., 2001). Our results show that treatment with TAD, HCQ, or their combination significantly increased Akt phosphorylation at Thr308 (Fig. 7, A and B), suggesting enhanced cell survival signaling. Insulin has been reported to protect against I/R injury via facilitating glucose transport (Oates et al., 2009), inhibition of apoptosis and inflammation (Sack and Yellon, 2003), and suppression of reactive oxygen species (Ji, et al., 2010). In the present study, we observed increased ATP production (Fig. 8), which is indicative of better fuel supply or improved mitochondrial biogenesis in the TAD and HCQ combination-treated hearts. In fact, a previous study showed that insulin and IGF-1 improved mitochondrial function through PI3K/Akt pathway in Huntington disease knock-in striatal cells (Ribeiro et al., 2014). The present data support similar observations in the diabetic mouse heart. Future studies are needed to elucidate the exact roles of mitochondrial respiratory chain and biogenesis in the TAD + HCQ-induced enhancement of cardiac ATP production.

It has been shown that preconditioning with IGF-1 protects against I/R injury (Buerke et al., 1995). In addition, IGF-1 exerts its indirect cardioprotective effect by increasing insulin sensitivity in the peripheral system (Abbas et al., 2008). Moreover, the cardioprotective effect of insulin and IGF-1 involves activation of biogenesis. The insulin/IGF-1- AktmTORC1 pathway regulates protein synthesis through activation of p70S6K and its target S6. There are two mTOR complexes: Raptor binding mTORC1, which is sensitive to nutrient availability and regulates cell cycle and proliferation; and Rictor binding mTORC2, which is rapamycin-insensitive and regulates cell survival in response to growth factors (Lum et al., 2005). In the present study, combination treatment with TAD- and HCQ-activated (phosphorylated) mTOR, Akt, and $\mathrm{S} 6$ after I/R, indicating an increased biogenesis in response to the energy restoration.

We also observed activation of mTORC2 in mice treated with either HCQ or the combination of TAD and HCQ. mTORC2 is insensitive to acute rapamycin treatment; however, it responds to growth factor, such as insulin signaling to regulate cell growth and survival (Lamming et al., 2012; Laplante and Sabatini, 2012). So far, the function of mTORC2 
A
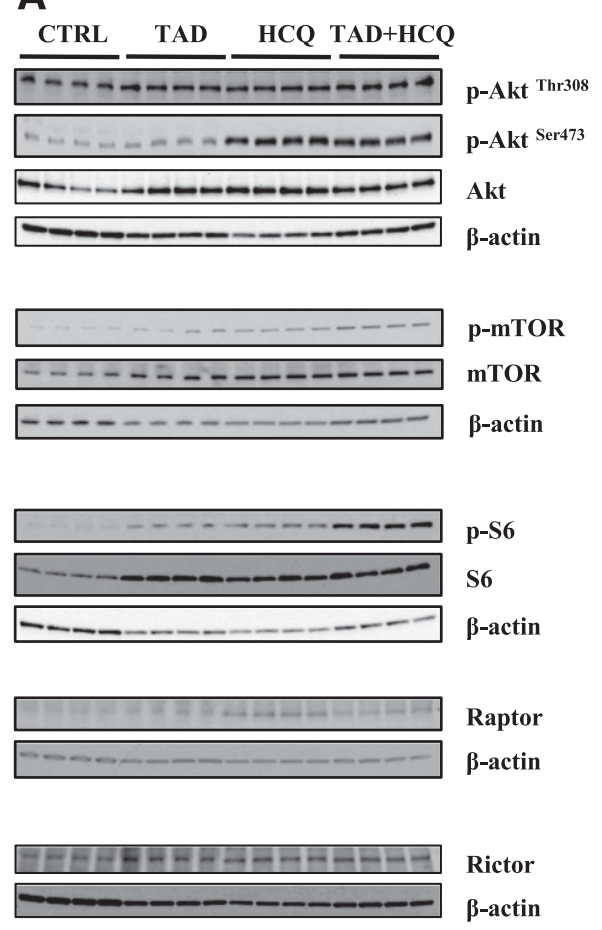

B

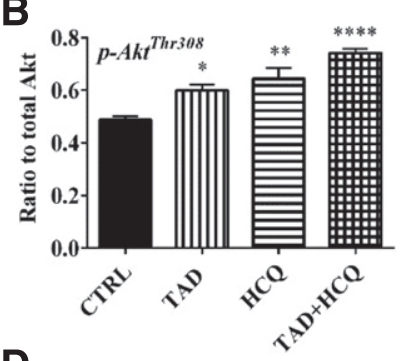

D

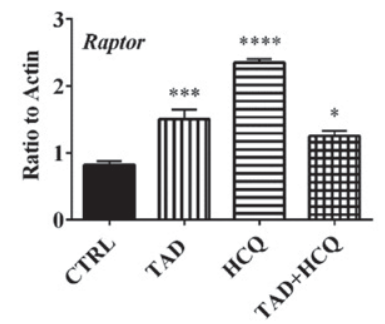

F

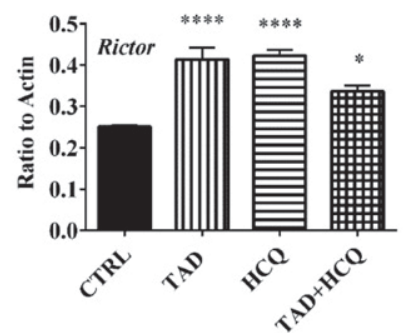

C

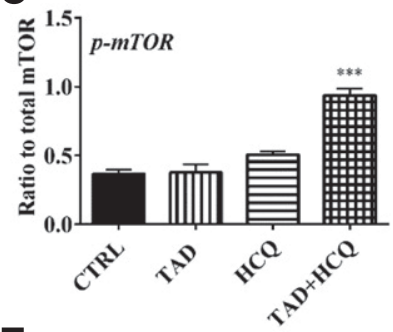

E

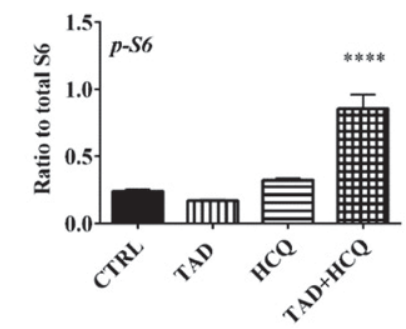

G

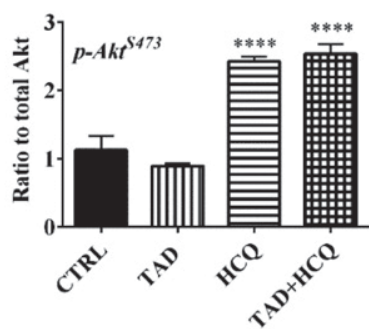

Fig. 7. Effect of TAD, HCQ, or combination treatment on mTOR activation after I/R. (A) Representative Western blot images; (B) bar diagram showing

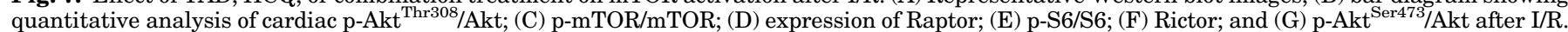
Data are presented as mean \pm S.E.M. $\left(n=4\right.$ /group). ${ }^{*} P<0.05 ; * * P<0.01 ; * * * P<0.001 ; * * * * P<0.0001$ versus CTRL group.

has not been fully uncovered. The most studied function of this complex is the phosphorylation of Akt at Ser473, leading to the full activation of the kinase. Our results show that Akt was highly phosphorylated at Ser473 after treatment with either HCQ or the combination of TAD and HCQ after I/R, indicating an increased activity of mTORC2 pathway.

Nevertheless, the present study has several limitations. First, we did not measure cardiac ATP levels during or after

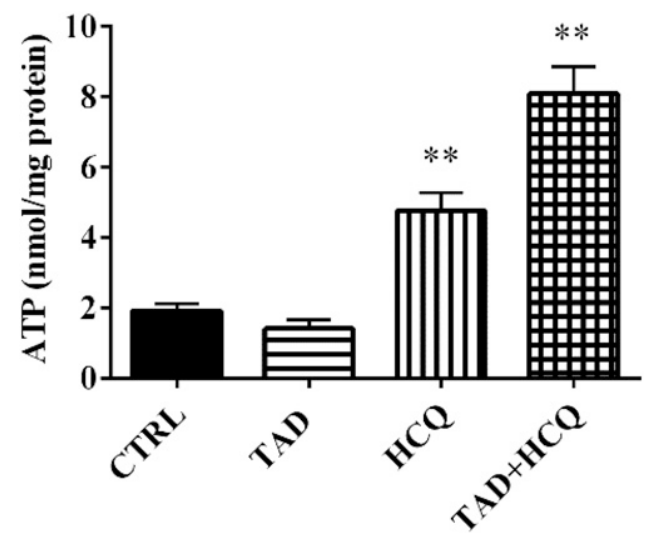

Fig. 8. Effect of TAD, HCQ, and combination treatment on myocardial ATP production. ATP levels are normalized with respective protein concentration for each sample. Data are presented as mean \pm S.E.M. $(n=$ 4/group). ${ }^{* *} P<0.01$ versus CTRL group. the global $\mathrm{I} / \mathrm{R}$ protocol because of the limited availability of post-I/R heart tissues that were used for infarct size measurement and molecular studies. Therefore, we speculate that possible changes in cardiac glucose and fatty acid metabolism after TAD + HCQ treatment may contribute to its infarctlimiting cardioprotective effects observed in the current study. The energy metabolism hypothesis needs to be tested in the in vivo $\mathrm{I} / \mathrm{R}$ model of diabetic conditions. Second, neither physiologic nor molecular studies were performed in the nonischemic isolated hearts receiving time-matched normoxic buffer-perfusion as the model-specific controls. In this case, any confounding effect attributable to heart isolation and buffer-perfusion procedures cannot be ruled out.

In summary, our results clearly demonstrate that shortterm treatment with the combination of TAD and HCQ significantly reduced myocardial infarct size after I/R in diabetic mice. Fasting glucose levels, as well as circulating levels of plasma free fatty acids and triglycerides, were decreased after combination treatment. Moreover, TAD and HCQ treatment resulted in better insulin sensitivity and higher baseline insulin levels, which were associated with larger pancreatic $\beta$-cell area and pancreas mass. These results suggest that combination treatment with TAD and HCQ could potentially be a novel therapy for both reducing cardiovascular risk factors and protecting against myocardial $\mathrm{I} / \mathrm{R}$ injury in T2D. We believe that treatment with TAD and HCQ could potentially lead to a novel line of therapy in diabetes clinics to manage cardiovascular risk factors and to improve clinical 
outcomes of diabetic patients suffering from acute myocardial infarction or possibly other ischemic injuries. Uniquely, this drug combination may also be beneficial in alleviating other common diabetic comorbidities, such as erectile dysfunction and nerve or joint pain, through the current Food and Drug Administration-approved drug indications for TAD and HCQ.

\section{Acknowledgments}

The authors thank Dr. Jorge A. Almenara, Department of Pathology, Virginia Commonwealth University, for helping with imaging the pancreas (H\&E-stained whole-slide picture).

\section{Authorship Contributions}

Participated in research design: Wang, Xi, Kukreja.

Conducted experiments: Wang, Xi.

Performed data analysis: Wang, Xi, Kukreja.

Wrote or contributed to the writing of the manuscript: Wang, $\mathrm{Xi}$, Kukreja.

\section{References}

Abbas A, Grant PJ, and Kearney MT (2008) Role of IGF-1 in glucose regulation and cardiovascular disease. Expert Rev Cardiovasc Ther 6:1135-1149.

Anderson RJ (1995) Hydroxychloroquine therapy in rheumatic diseases. Bull Rheum Dis 44:6-7.

Barsotti A, Giannoni A, Di Napoli P, and Emdin M (2009) Energy metabolism in the normal and in the diabetic heart. Curr Pharm Des 15:836-840.

Bili A, Sartorius JA, Kirchner HL, Morris SJ, Ledwich LJ, Antohe JL, Dancea S, Newman ED, and Wasko MC (2011) Hydroxychloroquine use and decreased risk of diabetes in rheumatoid arthritis patients. J Clin Rheumatol 17:115-120.

Blazar BR, Whitley CB, Kitabchi AE, Tsai MY, Santiago J, White N, Stentz FB, and Brown DM (1984) In vivo chloroquine-induced inhibition of insulin degradation in a diabetic patient with severe insulin resistance. Diabetes 33: 1133-1137.

Bolli R, Hartley CJ, and Rabinovitz RS (1991) Clinical relevance of myocardial "stunning". Cardiovasc Drugs Ther 5:877-890.

Bourke L, McCormick J, Taylor V, Pericleous C, Blanchet B, Costedoat-Chalumeau N, Stuckey D, Lythgoe MF, Stephanou A, and Ioannou Y (2015) Hydroxychloroquine Protects against Cardiac Ischaemia/Reperfusion Injury In Vivo via Enhancement of ERK1/2 Phosphorylation. PLoS One 10:e0143771.

Buerke M, Murohara T, Skurk C, Nuss C, Tomaselli K, and Lefer AM (1995) Cardioprotective effect of insulin-like growth factor I in myocardial ischemia followed by reperfusion. Proc Natl Acad Sci USA 92:8031-8035.

Das A, Durrant D, Salloum FN, Xi L, and Kukreja RC (2015) PDE5 inhibitors as therapeutics for heart disease, diabetes and cancer. Pharmacol Ther 147: $12-21$.

Das A, Salloum FN, Xi L, Rao YJ, and Kukreja RC (2009) ERK phosphorylation mediates sildenafil-induced myocardial protection against ischemia-reperfusion injury in mice. Am J Physiol Heart Circ Physiol 296:H1236-H1243.

Das A, Xi L, and Kukreja RC (2005) Phosphodiesterase-5 inhibitor sildenafil preconditions adult cardiac myocytes against necrosis and apoptosis. Essential role of nitric oxide signaling. J Biol Chem 280:12944-12955.

Fukushima A, Milner K, Gupta A, and Lopaschuk GD (2015) Myocardial energy substrate metabolism in heart failure: from pathways to therapeutic targets. Curr Pharm Des 21:3654-3664.

Galiè N, Brundage BH, Ghofrani HA, Oudiz RJ, Simonneau G, Safdar Z, Shapiro S, White RJ, Chan M, Beardsworth A, et al.; Pulmonary Arterial Hypertension and Response to Tadalafil (PHIRST) Study Group (2009) Tadalafil therapy for pulmonary arterial hypertension. Circulation 119:2894-2903.

Gilbert RE and Krum H (2015) Heart failure in diabetes: effects of antihyperglycaemic drug therapy. Lancet 385:2107-2117.

Ginter E and Simko V (2012) Type 2 diabetes mellitus, pandemic in 21st century. Adv Exp Med Biol 771:42-50.

Guler HP, Zapf J, and Froesch ER (1987) Short-term metabolic effects of recombinant human insulin-like growth factor I in healthy adults. $N$ Engl $J$ Med 317:137-140. Hage MP, Al-Badri MR, and Azar ST (2014) A favorable effect of hydroxychloroquine on glucose and lipid metabolism beyond its anti-inflammatory role. Ther Adv Endocrinol Metab 5:77-85.

Halaby MJ, Kastein BK, and Yang DQ (2013) Chloroquine stimulates glucose uptake and glycogen synthase in muscle cells through activation of Akt. Biochem Biophys Res Commun 435:708-713.

Hemnes AR and Champion HC (2006) Sildenafil, a PDE5 inhibitor, in the treatment of pulmonary hypertension. Expert Rev Cardiovasc Ther 4:293-300.

Hill KD, Eckhauser AW, Marney A, and Brown NJ (2009) Phosphodiesterase 5 inhibition improves beta-cell function in metabolic syndrome. Diabetes Care $\mathbf{3 2}$ 857-859.

Hügl SR, White MF, and Rhodes CJ (1998) Insulin-like growth factor I (IGF-I)stimulated pancreatic beta-cell growth is glucose-dependent. Synergistic activation of insulin receptor substrate-mediated signal transduction pathways by glucose and IGF-I in INS-1 cells. J Biol Chem 273:17771-17779.

Jenkins DP, Pugsley WB, and Yellon DM (1995) Ischaemic preconditioning in a model of global ischaemia: infarct size limitation, but no reduction of stunning. $J$ Mol Cell Cardiol 27:1623-1632.
Ji L, Fu F, Zhang L, Liu W, Cai X, Zhang L, Zheng Q, Zhang H, and Gao F (2010) Insulin attenuates myocardial ischemia/reperfusion injury via reducing oxidative/ nitrative stress. Am J Physiol Endocrinol Metab 298:E871-E880.

Jonassen AK, Sack MN, Mjøs OD, and Yellon DM (2001) Myocardial protection by insulin at reperfusion requires early administration and is mediated via Akt and p70s6 kinase cell-survival signaling. Circ Res 89:1191-1198.

Kim W, Doyle ME, Liu Z, Lao Q, Shin YK, Carlson OD, Kim HS, Thomas S, Napora JK, Lee EK, et al. (2011) Cannabinoids inhibit insulin receptor signaling in pancreatic $\beta$-cells. Diabetes 60:1198-1209.

Koka S, Aluri HS, Xi L, Lesnefsky EJ, and Kukreja RC (2014) Chronic inhibition of phosphodiesterase 5 with tadalafil attenuates mitochondrial dysfunction in type 2 diabetic hearts: potential role of NO/SIRT1/PGC-1 $\alpha$ signaling. Am J Physiol Heart Circ Physiol 306:H1558-H1568.

Kulkarni RN, Holzenberger M, Shih DQ, Ozcan U, Stoffel M, Magnuson MA and Kahn CR (2002) beta-cell-specific deletion of the Igf1 receptor leads to hyperinsulinemia and glucose intolerance but does not alter beta-cell mass. Nat Genet 31:111-115.

Lamming DW, Ye L, Katajisto P, Goncalves MD, Saitoh M, Stevens DM, Davis JG Salmon AB, Richardson A, Ahima RS, et al. (2012) Rapamycin-induced insulin resistance is mediated by mTORC2 loss and uncoupled from longevity. Science $\mathbf{3 3 5}$ $1638-1643$

Laplante M and Sabatini DM (2012) mTOR signaling in growth control and disease. Cell 149:274-293.

Long L, Yang X, Southwood M, Lu J, Marciniak SJ, Dunmore BJ, and Morrell NW (2013) Chloroquine prevents progression of experimental pulmonary hypertension via inhibition of autophagy and lysosomal bone morphogenetic protein type II receptor degradation. Circ Res 112:1159-1170.

Lu Y, Herrera PL, Guo Y, Sun D, Tang Z, LeRoith D, and Liu JL (2004) Pancreaticspecific inactivation of IGF-I gene causes enlarged pancreatic islets and significant resistance to diabetes. Diabetes 53:3131-3141.

Lum JJ, Bauer DE, Kong M, Harris MH, Li C, Lindsten T, and Thompson CB (2005) Growth factor regulation of autophagy and cell survival in the absence of apoptosis. Cell 120:237-248.

Mansor LS, Mehta K, Aksentijevic D, Carr CA, Lund T, Cole MA, Le Page L, Sousa Fialho MdaL, Shattock MJ, Aasum E, et al. (2016) Increased oxidative metabolism following hypoxia in the type 2 diabetic heart, despite normal hypoxia signalling and metabolic adaptation. $J$ Physiol 594:307-320.

Miettinen H, Lehto S, Salomaa V, Mähönen M, Niemelä M, Haffner SM, Pyörälä K, and Tuomilehto J; The FINMONICA Myocardial Infarction Register Study Group (1998) Impact of diabetes on mortality after the first myocardial infarction. $\mathrm{Di}$ abetes Care 21:69-75.

Moelker AD, Baks T, van den Bos EJ, van Geuns RJ, de Feyter PJ, Duncker DJ, and van der Giessen WJ (2006) Reduction in infarct size, but no functional improvement after bone marrow cell administration in a porcine model of reperfused myocardial infarction. Eur Heart $J$ 27:3057-3064.

Morand EF, McCloud PI, and Littlejohn GO (1992) Continuation of long term treatment with hydroxychloroquine in systemic lupus erythematosus and rheumatoid arthritis. Ann Rheum Dis 51:1318-1321.

Nichols GA, Joshua-Gotlib S, and Parasuraman S (2013) Glycemic control and risk of cardiovascular disease hospitalization and all-cause mortality. J Am Coll Cardiol 62:121-127.

Oates A, Nubani R, Smiley J, Kistler L, Hughey S, Theiss P, Perez-Tamayo RA, Eiferman D, Lonchyna V, and Higgins RS (2009) Myocardial protection of insulin and potassium in a porcine ischemia-reperfusion model. Surgery 146: $23-30$.

Ockaili R, Salloum F, Hawkins J, and Kukreja RC (2002) Sildenafil (Viagra) induces powerful cardioprotective effect via opening of mitochondrial $\mathrm{K}_{\text {(ATP) }}$ channels in rabbits. Am J Physiol Heart Circ Physiol 283:H1263-H1269.

Powrie JK, Shojaee-Moradie F, Watts GF, Smith GD, Sönksen PH, and Jones RH (1993) Effects of chloroquine on the dyslipidemia of non-insulin-dependent diabetes mellitus. Metabolism 42:415-419.

Ribeiro M, Rosenstock TR, Oliveira AM, Oliveira CR, and Rego AC (2014) Insulin and IGF-1 improve mitochondrial function in a PI-3K/Akt-dependent manner and reduce mitochondrial generation of reactive oxygen species in Huntington's disease knock-in striatal cells. Free Radic Biol Med 74:129-144.

Sack MN and Yellon DM (2003) Insulin therapy as an adjunct to reperfusion after acute coronary ischemia: a proposed direct myocardial cell survival effect independent of metabolic modulation. J Am Coll Cardiol 41:1404-1407.

Salloum F, Yin C, Xi L, and Kukreja RC (2003) Sildenafil induces delayed preconditioning through inducible nitric oxide synthase-dependent pathway in mouse heart. Circ Res 92:595-597.

Salloum FN, Chau VQ, Hoke NN, Abbate A, Varma A, Ockaili RA, Toldo S, and Kukreja RC (2009) Phosphodiesterase-5 inhibitor, tadalafil, protects against myocardial ischemia/reperfusion through protein-kinase g-dependent generation of hydrogen sulfide. Circulation 120(11, Suppl)S31-S36.

Selvaraj P and Pugalendi KV (2012) Efficacy of hesperidin on plasma, heart and liver tissue lipids in rats subjected to isoproterenol-induced cardiotoxicity. Exp Toxicol Pathol 64:449-452.

Sisó A, Ramos-Casals M, Bové A, Brito-Zerón P, Soria N, Muñoz S, Testi A, Plaza J, Sentís J, and Coca A (2008) Previous antimalarial therapy in patients diagnosed with lupus nephritis: influence on outcomes and survival. Lupus 17: $281-288$

Stich V and Berlan M (2004) Physiological regulation of NEFA availability: lipolysis pathway. Proc Nutr Soc 63:369-374.

Varma A, Das A, Hoke NN, Durrant DE, Salloum FN, and Kukreja RC (2012) Antiinflammatory and cardioprotective effects of tadalafil in diabetic mice. PLoS One 7: e45243.

Wasko MC, McClure CK, Kelsey SF, Huber K, Orchard T, and Toledo FG (2015 Antidiabetogenic effects of hydroxychloroquine on insulin sensitivity and beta cell function: a randomised trial. Diabetologia 58:2336-2343. 
Xi L, Hess ML, and Kukreja RC (1998) Ischemic preconditioning in isolated perfused mouse heart: reduction in infarct size without improvement of post-ischemic ventricular function. Mol Cell Biochem 186:69-77.

Xi L, Jarrett NC, Hess ML, and Kukreja RC (1999) Essential role of inducible nitric oxide synthase in monophosphoryl lipid A-induced late cardioprotection: evidence from pharmacological inhibition and gene knockout mice. Circulation 99:2157-2163.

Xu Y, Ma LL, Zhou C, Zhang FJ, Kong FJ, Wang WN, Qian LB, Wang CC, Liu XB, Yan M, et al. (2013) Hypercholesterolemic myocardium is vulnerable to ischemiareperfusion injury and refractory to sevoflurane-induced protection. PLoS One 8 e76652.
Yellon DM and Hausenloy DJ (2007) Myocardial reperfusion injury. $N$ Engl J Med 357:1121-1135.

Address correspondence to: Dr. Rakesh C. Kukreja, Scientific Director, Pauley Heart Center, Professor of Medicine, Physiology, Biochemistry, Division of Cardiology, Box 980204, Virginia Commonwealth University, 1101 East Marshall Street, Room 7-020D, Richmond, VA 23298-0204. E-mail: rakesh.kukreja@vcuhealth.org 